\title{
MedienPädagogik
}

Zeitschrift für Theorie und Praxis der Medienbildung

Jahrbuch Medienpädagogik 4.

Zweitveröffentlichung aus: Jahrbuch Medienpädagogik 4. (2005) Wiesbaden: VS Verlag für Sozialwissenschaften. Hrsg. v. Ben Bachmair, Peter Diepold und Claudia de Witt.

\section{Hauptkomponentenanalytische Untersuchungen zum Medienhandeln Jugendlicher}

\author{
Klaus Peter Treumann, Eckhard Burkatzki, Mareike Strotmann und Claudia Wegener
}

Die vorliegende Arbeit ist im Rahmen des von der DFG geförderten Forschungsprojekts „Eine Untersuchung zum Mediennutzungsverhalten 12-20jähriger und zur Entwicklung von Medienkompetenz im Jugendalter“ entstanden, welches von Klaus Peter Treumann, Uwe Sander und Dorothee Meister geleitet wird. Es bezieht sich auf die Bundesländer Mecklenburg-Vorpommern, Nordrhein-Westfalen und Sachsen-Anhalt mit den drei Projektstandorten Bielefeld, Halle a.d. Saale und Rostock. Das Forschungsvorhaben kombiniert qualitative und quantitative Zugänge zum Forschungsfeld in Form von Gruppendiskussionen, leitfadengesteuerten Interviews und einer standardisierten Befragung von Jugendlichen. Im Folgenden wird über einige Befunde quantitativer Analysen berichtet.

\section{Fragestellung}

Ein Ziel des quantitativen Teils des Forschungsprojekts ist es, ein möglichst umfassendes Bild über das Medienhandeln Jugendlicher zu gewinnen, d.h. zu repräsentativen Aussagen über die Häufigkeitsverteilung quantitativ erfassbare Merkmale im Kollektiv der 12- bis 20-Jährigen zu gelangen. Medienhandeln umfasst dabei nach unserem Verständnis nicht nur Variablen der Mediennutzung, sondern schließt darüber hinaus solche Konzepte ein, wie sie in verschiedenen systematischen Entwürfen zur Medienkompetenz entwickelt worden sind. Als theoretische Folie nutzen wir vor allem das Bielefelder Medienkompetenz-Modell, um das Medienhandeln Jugendlicher zum einen facettenreich zu operationalisieren und zum anderen inhaltlich in voneinander unterscheidbare Teilbereiche zu untergliedern. Die quantitative Studie bleibt jedoch nicht bei einer uni- und bivariaten Analyse stehen, sondern versucht explorativ mit Hilfe des multivariaten Verfahrens der Hauptkomponentenanalyse die folgenden Fragen zu beantworten:

a) Lassen sich die erfassten (,gemessenen“) Variablen des Medienhandelns Jugendlicher gemäß ihrer korrelativen Beziehungen in voneinander unabhängigen Gruppen klassifizieren? Es geht also darum, die Hauptquel- 
len der Variation bzw. der Unterschiede, welche die Jugendlichen in ihren Medienaktivitäten zeigen, zu rekonstruieren.

b) Wie sind die einzelnen Teilbereiche des Medienhandelns dimensioniert? Ist die dem Variablengeflecht unterlegte Ordnung, aus der sich die jeweils angetroffene Konstellation der Variableninterkorrelationen erklären lässt, ein- oder mehrdimensional? In diesem Zusammenhang lassen sich auch Überlegungen zur Konstruktvalidität von Merkmalsbereichen diskutieren.

\section{Theoretischer Bezugsrahmen}

Im Folgenden werden einige Theoriebezüge, die für die Operationalisierung der Variablen der quantitativen Untersuchung und für die Interpretation der Ergebnisse der Hauptkomponentenanalysen von Bedeutung sind, knapp dargestellt.

\subsection{Das Bielefelder Medienkompetenz-Modell}

Medienkompetenz ist eine Teilmenge der kommunikativen Kompetenz, die sich zur Realisierung von Kommunikation vielfältiger Medien bedient. Das Bielefelder Medienkompetenz-Modell (vgl. Baacke 1996, 1999) umfasst vier Dimensionen mit jeweils mehreren Unterdimensionen, um Reichweite und Umfang medial vermittelter Kommunikation zu rekonstruieren:

\section{Dimension: Mediennutzung}

Sie lässt sich in zwei Subdimensionen ausdifferenzieren:

a) Die rezeptiv-anwendende Unterdimension bezeichnet die Fähigkeit, das Gelesene, das Gehörte oder das Gesehene zu verarbeiten und in das Bildungs- und Bildrepertoire einzuarbeiten.

b) Die zweite Unterdimension umfasst die interaktive Nutzung von Medien: Hier ist der Nutzer nicht nur Rezipient, sondern im Rahmen der Kommunikationssituation auch Anbieter von Medienbotschaften. Hierzu gehört die Fähigkeit, nach medienvermittelten Informationen zu fragen oder umgekehrt auf Anfragen antworten zu können, wie sie z.B. bei der Informationsrecherche mit Hilfe von Suchmaschinen, beim Telebanking, Teleshopping oder beim Online-Learning notwendig ist, aber auch beim Produzieren eines Videos in einer Jugendgruppe.

\section{Dimension: Medienkunde}

Sie bezieht sich auf das Wissen über heutige Mediensysteme und -strukturen:

a) Die informative Subdimension umfasst klassische Wissensbestände (Medienwissen). Hierzu gehört beispielsweise das Wissen um die Strukturen 
des dualen Rundfunksystems, um die Arbeit von Journalisten, um Programmformate und -genres sowie Wissen über den effektiven Einsatz des Computers als Arbeitshilfe.

b) Die instrumentell-qualifikatorische Unterdimension Unterdimension meint hingegen die Fähigkeit, mit Medien angemessen umzugehen. Dazu gehört etwa das Sich-Einarbeiten in die Handhabung einer Computer-Software, das Sich-Einloggen-Können in ein Netz, die Bedienung des Videorecorders.

\section{Dimension: Mediengestaltung}

Sie bezieht sich auf den Prozess der technischen und inhaltlichen Veränderung von Medien und Medienangeboten. Auch hier lassen sich zwei Unterdimensionen konstruieren:

a) Die innovative im Sinne von Veränderungen und Weiterentwicklungen des Mediensystems innerhalb der angelegten Logik und

b) die kreative im Sinne einer Betonung ästhetischer Varianten, das Über- die-Grenzender-Kommunikationsroutine-Gehen (z.B. das Verfremden bekannter Werbelogos zum Zweck der Ironisierung oder Provokation). Diese Unterdimension schließt ebenso die Produktion von Medienbotschaften ein, wie etwa die Produktion von Femsehbeiträgen für den Offenen Kanal oder die Gestaltung einer Homepage.

\section{Dimension: Medienkritik}

Sie umfasst drei unterschiedliche Aspekte:

a) Analytisch sollen problematische Prozesse (z.B. Konzentrationsbewegungen im Mediensektor) angemessen erfasst werden können.

b) Die reflexive Unterdimension zielt auf den Gedanken, dass jeder Mensch sein analytisches und sonstiges Wissen auf sich selbst und sein persönliches Handeln beziehen und anwenden können muss.

c) Die ethische Unterdimension bindet analytisches Denken und reflexive Bezüge in ethische Konzepte ein und definiert sie somit als sozial verantwortlich (z.B. Fragen der gesellschaftlichen Folgen medialer Entwicklungen).

\subsection{Der Uses-and-Gratifications Approach}

Der Nutzenansatz fragt nach den Gründen der Mediennutzung und dem Nutzen der durch die Medien vermittelten Inhalte für den Rezipienten. Er begreift den Mediennutzer als Subjekt, das medial vermittelte Realität produktiv verarbeitet. Der Uses-and-Gratifications Approach versteht sich als bewusste Abkehr von den Annahmen des StimulusResponse-Modells, indem er die aktive Rolle des Publikums in den Mittelpunkt des Forschungsinteres- 
ses stellt (vgl. Katz/Blumler 1974). Mehr als Wirkungen interessieren im Rahmen dieses Ansatzes Funktionen der Medien für den Rezipienten.

Der Uses-and-Gratifications Approach bekommt durch die Einführung Neuer Medien neue und umfassende Perspektiven. So steigt das Aktivitätspotential des Rezipienten ja zum einen durch die Interaktivität multimedialer Anwendungsmöglichkeiten, zum anderen durch den Abbau räumlicher, zeitlicher und funktionaler Einschränkungen bei der Mediennutzung. Insofern bietet sich dieser Ansatz insbesondere auch als Grundlage zur Untersuchung des Medienverhaltens im Hinblick auf die Neuen Medien an.

\subsection{Habituskonzept und Kapitalsortenansatz}

Das Habituskonzept von Bourdieu einschließlich seines Kapitalsortenansatzes, welches Habitus als ein durch Lernprozesse inkorporiertes Dispositionssystem begreift, das Wahmehmungs-, Denk- und Handlungsschemata bereit hält, die wiederum Orientierungen innerhalb der sozialen Welt ermöglichen und die der Hervorbringung angemessener Praktiken dienen. Das Ausmaß an ökonomischem, kulturellem und sozialem Kapital, über das der einzelne verfügen kann, ist zentrale Determinante des Erwerbs und der Ausübung von Kompetenzen.

Die Hinwendung zu Jugendkulturen (und den entsprechenden Medien, die sie begleiten) ist zu verstehen als ein Akt der Selbstsozialisation Jugendlicher. Die unterschiedliche Verfügung über kulturelles Kapital bedingt unterschiedliche Aneignungsformen (und Interessen) an kulturellen Angeboten, so dass sich die Differenzen weiter verstärken. Die Analogie zur Wissenskluftthese in der Medienforschung - enger bezogen auf den Erwerb von Wissen - ist evident.

Im Unterschied zum ökonomischen Kapital ist das kulturelle Kapital grundsätzlich körpergebunden und setzt Verinnerlichung voraus. Wer sich bildet, muss zunächst Zeit und Lernanstrengungen aufwenden, um inkorporiertes Kapital als ein Besitztum zu erwerben, das dadurch zu einem festen Bestandteil der Person, zum Habitus geworden ist. „Inkorporiertes und damit verinnerlichtes Kapital kann deshalb (im Unterschied zu Geld, Besitz- und Adelstiteln) nicht durch Schenkung, Vererbung, Kauf und Tausch kurzfristig weitergeben werden“ (Bourdieu 1997, S. 56). Die sozialen Bedingungen der Weitergabe kulturellen Kapitals sind verborgener als beim ökonomischen Kapital, sie lassen sich dennoch nachzeichnen. Der Besitz kulturellen Kapitals ist über den subjektiven Gewinn hinaus, besser gebildet zu sein, auch deshalb vorteilhaft, weil es für materielle und symbolische Profite eingesetzt werden kann. Wer beispielsweise über Medienkompetenz als kulturelles Kapital verfügt, ist in der Lage, Medien anders, nämlich besser, für seine Zwecke und Interessen einsetzen - privat und/oder beruflich. Kulturelles Kapital ermöglicht dem Einzelnen so einen Zugang zu Statusgewinnen (jemand, der sich mit Neuen Medien auskennt) oder auch ökonomischen Gewinnen 
(Computerkenntnisse als Voraussetzung für Berufspositionen) in seinem gesellschaftlichen Umfeld.

Mit sozialem Kapital bezeichnet Bourdieu „die Gesamtheit der aktuellen und potenziellen Ressourcen, die mit dem Besitz eines dauerhaften Netzes von mehr oder weniger institutionalisierten Beziehungen gegenseitigen Rennens oder Anerkennens verbunden sind; oder, anders ausgedrückt, es handelt sich dabei um Ressourcen, die auf der Zugehörigkeit zu einer Gruppe beruhen. (...) Sozialkapitalbeziehungen können nur in der Praxis auf der Grundlage von materiellen und/oder symbolischen Tauschbeziehungen existieren, zu deren Aufrechterhaltung sie beitragen“ (Bourdieu 1997, S. 63). Wer über tragfähige Netze im Familien-, Freundes- und Kollegenkreis verfügt, kann auch an deren Ressourcen partizipieren, so dass dem Sozialkapital ein besonderer Multiplikatoreffekt zu eigen ist. Soziales und kulturelles Kapital sind bereits beim Erwerb von Medienkompetenz entscheidende Faktoren. Ein Mangel an diesen Kapitalsorten kann den Erwerb von Medienkompetenz behindern (vgl. Treumann u.a. 2002).

\section{Forschungsdesign}

Der Erhebungsplan für die standardisierte Befragung der Jugendlichen zu ihrem Medienhandeln ist als Querschnittsstudie angelegt, d.h. die Datenerhebung bezieht sich auf eine kurze Zeitspanne, in der eine einmalige Erhebung der Variablenwerte bei n Untersuchungseinheiten vorgenommen wird (Diekmann 1995, S. 267).

\subsection{Stichprobe}

Die Grundgesamtheit setzt sich aus Jugendlichen der drei Bundesländer MecklenburgVorpommern, Nordrhein-Westfalen und Sachsen-Anhalt zusammen, die zum Befragungszeitpunkt ein Lebensalter von 12 bis einschließlich 20 Jahren erreicht hatten, Deutsch als Muttersprache beherrschten, mindestens drei Jahre in demselben Ort und jeweils in einem der drei folgenden Sozialräume wohnten:

a) Ländliche Wohngegenden mit einer Gesamteinwohnerzahl bis 25.000

b) Mittelstädte mit einer Einwohnerzahl bis 50.000 und

c) Großstädte ab einer Einwohnerzahl von 100.000

Aus der Grundgesamtheit wurde eine zweifach geschichtete Flächenstichprobe gezogen, die sich zum einen auf die drei Bundesländer und zum anderen auf die drei Sozialräume bezog. In einem letzten Schritt wurde in den drei ausgewählten Sozialräumen per Zufall mindestens je eine Hauptschule, Realschule bzw. Sekundarschule, ein Gymnasium, eine Gesamtschule (für NRW) und eine 
Berufsschule ausgewählt und die Jugendlichen aus mindestens einer Schulklasse pro Schülerjahrgang von der siebenten Jahrgangsstufe oder dem ersten Ausbildungsjahr an aufwärts befragt. Es ergab sich über alle drei Bundesländer hinweg eine bereinigte Nettostichprobe von insgesamt $n=3.271$ Jugendlichen. Diese Stichprobe bildet den Ausgangspunkt für die nachfolgenden Analysen.

\subsection{Methoden der Datenerhebung}

Die Umfrage zum Mediennutzungsverhalten und zur Medienkompetenz Jugendlicher erfolgte mit Hilfe eines standardisierten Fragebogens, der insgesamt 121 Fragen umfasste und zuvor einem Pretest unterzogen wurde. Die Operationalisierung des Medienhandelns erfolgte u.a. in Anlehnung an die Studien von Gerhards und Klingler (2001). Der Erhebungszeitraum in den drei Bundesländern erstreckte sich für die Haupterhebung von Mitte Oktober bis Anfang Dezember 2001.

\section{Methoden der Datenanalyse}

\subsection{Itemanalyse}

Zur Messung der Unterdimension „informative Medienkunde“ (Medienwissen) des Bielefelder Medienkompetenz-Modells bildeten wir für jeden Jugendlichen einen Summenscore bzw. Index aus den richtig beantworteten Einzelitems (für jedes Item galt: richtig $=1$ Punkt; falsch $=0$ Punkte). Zuvor unterzogen wir die entsprechenden Items einer Itemanalyse: Ausgeschieden wurden zum Einen alle Items, die sehr schwierig $\left(\mathrm{p}_{\mathrm{i}}\right.$ $<0,2)$ oder sehr leicht $\left(\mathrm{p}_{\mathrm{i}}>0,8\right)$ waren, und zum anderen diejenigen, deren Trennschärfekoeffizient $r_{i t}<0,25$ war (Bortz u. Döring $2002^{3}$, S. 218-219). Die Items, die nach Durchführung der Schwierigkeits- und Trennschärfenanalyse noch in der Untersuchung verblieben, bildeten den Ausgangspunkt für die Hauptkomponentenanalysen zur informativen Medienkunde beziehungsweise zum Medienwissen. Des Weiteren wurde die Reliabilität - in unserem Fall eines Index zur Messung des Medienwissens - als AlphaKoeffizient nach Cronbach berechnet (siehe Abschnitt 5.4).

\subsection{Durchführung der Hauptkomponentenanalysen}

Die Hauptkomponentenanalyse ${ }^{1}$ gehört zur Klasse der multivariaten Verfahren der Datenanalyse (vgl. Backhaus u.a. 2000; Bortz 1999; Cureton/D’Agostino 1983; Jackson 1991; Jolliffe 1986; Krzanowski/Marriott 1994 u. 1995; Ta-

1 Wir verwenden in dieser Veröffentlichung allein aus Gründen der sprachlichen Variation die Begriffe „Hauptkomponenten“ und „Faktoren“ synonym. 
bachnik/Fidell 1996). Sie ist trotz mancher Ähnlichkeiten von der statistischen Methode der Faktorenanalyse zu unterscheiden. In der Hauptkomponentenanalyse wird im Gegensatz zur Faktorenanalyse die gesamte Varianz einer Variablen, die durch die Standardisierung vom Betrag 1 ist, analysiert, d.h. es wird nicht zwischen gemeinsamer Varianz, spezifischer Varianz und Fehlervarianz der Variablen unterschieden. Die Hauptkomponenten, die inhaltlich ähnliche Merkmale zu Variablenkomplexen bündeln, werden durch eine lineare Transformation eines umfangreichen Sets korrelierter Variablen gewonnen und ergeben so eine kleine Gruppe unkorrelierter Variablenkomplexe (= die Komponenten). Bei diesem Verfahren schöpft die erste Komponente den größtmöglichen Varianzanteil aus, die zweite Komponente den größtmöglichen Anteil der dann noch verbliebenen Varianz (= Restvarianz) und so fort. Die Hauptkomponentenanalyse liefert Indexzahlen (sog. Ladungen), die darüber informieren, wie gut eine Variable zu einer Variablengruppe, d.h. zu einer Komponente passt. Diese Ladungen der Variablen auf der jeweiligen Hauptkomponente stellen die Basis für interpretative Hypothesen über das Gemeinsame der Variablen einer Variablengruppe dar.

\subsubsection{Bestimmung der Anzahl der zu extrahierenden Hauptkomponenten}

Für jede Unterdimension des Bielefelder Medienkompetenz-Modells - z.B. interaktive Mediennutzung - wurde zwischen den entsprechenden Variablen eine Korrelationsmatrix errechnet und mit Hilfe der Hauptkomponentenanalyse exploriert. Das Verfahren extrahierte in einem ersten Schritt aus der Korrelationsmatrix eine Reihe von Hauptkomponenten. Dabei galt es, die Anzahl der Komponenten so zu bestimmen, dass zum einen ein hinreichend großer Teil der Streuungen bzw. Varianz - in unserem Fall der Unterschiede zwischen den Jugendlichen in bestimmten Bereichen ihres Medienhandelns - erklärt wird und zugleich eine ausreichend große Reduzierung der Komplexität des untersuchten Bereichs erfolgt, d.h. dass die vielfältigen Medienaktivitäten der Jugendlichen, welche durch die einzelnen Items des Fragebogens erfasst wurden, sich zu sinnvoll interpretierbaren Klassen bzw. Kategorien zusammenfassen lassen. Diese Bündelung der Variablen zu wenigen Hauptkomponenten führt zu einer geringeren Varianzaufklärung als maximal möglich wäre. Sie wird zugunsten einer Reduktion einer komplexen Datenmenge bewusst in Kauf genommen (Prinzip der Parsimonität), und zwar unter der Annahme, dass der nicht-erklärte Varianzanteil auf zufällige oder irrelevante Einflüsse (sogenanntes „weißes Rauschen“) zurückgeht. Die Faktoren einer Hauptkomponentenanalyse sind voneinander unabhängig und erklären sukzessiv maximale Varianzanteile der analysierten Korrelationsmatrix.

Die Bestimmung der Anzahl der zu extrahierenden Hauptkomponenten erfolgte durch eine kombinierte Anwendung des Kaiser-Guttman-Kriteriums, d.h. nur Komponenten mit Eigenwerten $>1$ sind zu berücksichtigen, und des Scree-Tests nach Catell sowie nach inhaltlichen Gesichtspunkten, wie etwa Interpretierbarkeit, Kohärenz und Prägnanz der Komponenten. 


\subsubsection{Rotation der Hauptkomponenten}

In einem zweiten Arbeitsschritt wurden die extrahierten Hauptkomponenten - geometrisch als Achsen im Variablenraum deutbar - zu ihrer besseren Interpretierbarkeit mit Hilfe des Varimax-Kriteriums auf eine orthogonale Einfachstruktur hin rotiert. Dadurch lädt auf den einzelnen Hauptkomponenten jeweils nur eine begrenzte Anzahl von Variablen substantiell, was in der Regel ihre Deutung erleichtert (Bortz 19995 , S. 531). Da die Hauptkomponenten orthogonal zueinander stehen, dürfen die Faktorenladungen $\mathrm{a}_{\mathrm{ij}}$ als Korrelationskoeffizienten ${ }^{2}$ der Komponenten mit den Variablen interpretiert werden. Bei der inhaltlichen Interpretation der Hauptkomponenten wurden nur solche Variablen berücksichtigt, die substantielle Ladungen aufwiesen $\left(a_{i j}>|0,40|\right)$. Sie berücksichtigten wir werden in den folgenden Abbildungen der Komponentenstrukturen als Indikatorvariablen bezeichnet.

\section{Ergebnisse}

Aus Platzgründen beschränken wir uns auf die Darstellung der faktoriellen Binnenstruktur der „rezeptiven Mediennutzung“, der „interaktiven Medienutzung“, der „informativen Medienkunde“ und der „kreativen Mediengestaltung“.

\subsection{Rezeptive Mediennutzung}

Die rezeptive Mediennutzung der Schüler wurde auf Grundlage objektivierter Zeit- und Häufigkeitsangaben zur Nutzungsdauer verschiedener Medien sowie über Fragen zur subjektiven Häufigkeit bestimmter Formen des Mediengebrauchs erfasst.

\subsubsection{Indikatoren der rezeptiven Mediennutzung}

Bezogen auf die Nutzungsdauer von Medien wurden Jugendliche danach gefragt, wie viel Zeit sie mit der Nutzung eines Mediums am Tag oder in der Woche verbringen. Vorgegeben war dabei immer eine konkrete Zeiteinheit (z.B. Stunden am Tag), auf deren Grundlage die Befragten die eigene durchschnittliche Nutzungsdauer eines Mediums quantifizieren konnten. Die entsprechende Frage zum Fernsehen lautete etwa: „Wie viele Stunden siehst du am Tag Fern-

2 Der Produkt-Moment-Korrelationskoeffizient $r$ kann Werte zwischen -1 und +1 annehmen: $-1 \leq \mathrm{r} \leq+1$. Eine Korrelation von $\mathrm{r}=+1$ bedeutet einen perfekten positiven Zusammenhang zwischen Hauptkomponente und Variable, ein Wert von $\mathrm{r}=-1$ einen perfekten negativen Zusammenhang; ein Korrelationskoeffizient von Null $(\mathrm{r}=0)$ signalisiert dagegen, dass kein Zusammenhang zwischen Komponente und Variable besteht. 
sehen?“ Die Fragen zur Nutzungsdauer der anderen Medien hatten analoge Formulierungen. Für die Analyse der rezeptiven Mediennutzung wurden die Nutzungsdauern primär solcher Medien ausgewählt, deren Gebrauch typischerweise eher konsumtiv angelegt ist, wie etwa Fernsehen, Radio, Walk- respektive Discman, Video, Kino, Lektüre von Belletristik und Zeitschriften.

\subsubsection{Hauptkomponentenmodell zur rezeptiven Mediennutzung}

Auf Grundlage der Hauptkomponentenanalyse wurde im Weiteren geprüft, wie sich die faktorielle Binnenstruktur der rezeptiven Mediennutzung auf Grundlage der Befragtenangaben darstellt. Im Zuge der Analysen ergab sich, wie im Folgenden dargestellt, als beste Rekonstruktion der Daten ein Fünf- Komponenten-Modell, auf dessen Basis insgesamt 51,4 Prozent der Varianz in der den Analysen zugrundegelegten Korrelationsmatrix aufgeklärt werden konnte (siehe Abb. 5.1.2).

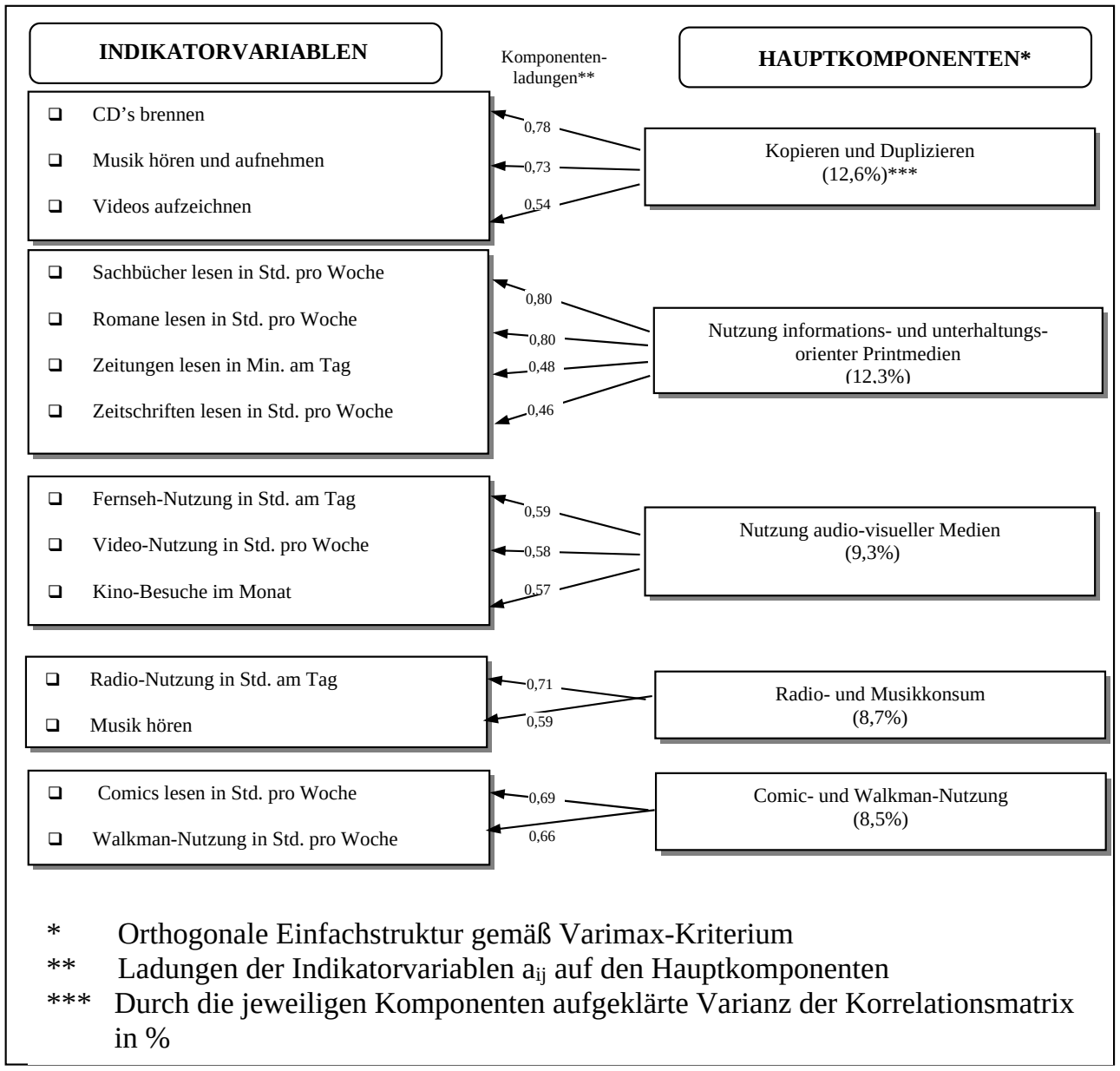

Abb. 5.1.2: Hauptkomponentenstruktur der rezeptiven Mediennutzung von Jugendlichen zwischen 12 und 20 Jahren $(n=1694)$. 


\section{Hauptkomponente 1: Kopieren und Duplizieren}

Die Komponente 1 vereinigt drei Items mit subjektiven Häufigkeitsangaben zum Kopieren und Duplizieren medialer Inhalte: Erstens das Item „CD’s brennen“, zweites „Musik hören und aufnehmen“ und drittens das Item „Videos aufzeichnen“. Dieser Befund lässt sich unter Rückbezug auf das quantitative Paradigma, welches von Auftrittshäufigkeiten von Merkmalen und ihren Ausprägungen ausgeht, wie folgt interpretieren: Bezogen auf das jugendliche Medienhandeln bedeutet dieser Befund, dass Jugendliche, die besonders häufig CD’s brennen - beispielsweise Musikstücke kopieren oder (neu) zusammenstellen -, auch überdurchschnittlich oft Musik hören und sie aufnehmen sowie Videos aufzeichnen. Wer dagegen nie oder selten CD’s brennt, der hört ebenfalls unterdurchschnittlich oft Musik, nimmt sie kaum oder gar nicht auf und stellt nie oder selten (Femseh-)Filme, Fernsehsendungen oder -spots per Video zusammen oder kopiert diese. Der Faktor ist interessanterweise die erklärungskräftigste Teilkomponente des Hauptkomponentenmodells und vereinigt, für sich betrachtet, 12,6 Prozent der Varianz in der zugrundeliegenden Korrelationsmatrix.

\section{Hauptkomponente 2: Nutzung informations- und unterhaltungsorientierter Printmedien}

Die Komponente 2 umfasst die rezeptive Nutzung von Printmedien. Auf ihr laden die Variablen zur Erfassung der objektiven Nutzungsdauer von belletristischer und sachbezogener Buchliteratur sowie von Zeitungen und Zeitschriften. Ihre besondere Prägnanz gewinnt diese Hauptkomponente durch das Lesen von Sachbüchern und Romanen, da diese beiden Merkmale jugendlichen Medienhandelns auf dem Faktor besonders hohe Ladungen aufweisen. Die Ladungen aller vier Items verfügen dabei über ein positives Vorzeichen, was anzeigt, dass durch die Komponente kein bipolares Kontinuum mit den Endpunkten einer unterhaltungs- versus informationsorientierten Rezeption von Printmedien rekonstruiert wird. Medial vermittelte Unterhaltungs- und Informationsorientierung scheinen hier vielmehr ineinander zu greifen. Die Hauptkomponente vereinigt dabei 12,3 Prozent der Varianz der zugrunde liegenden Korrelationsmatrix auf sich.

\section{Hauptkomponente 3: Nutzung audio-visueller Medien}

Komponente 3 bündelt drei Variablen zur individuellen Nutzungsdauer der Medien Fernsehen, Video und Kino. Auch hier gilt - wie prinzipiell bei allen Komponenten - gemäß dem quantitativen Paradigma, welches auf Häufigkeitsbesetzungen von Merkmalsausprägungen beruht, dass Jugendliche, die überdurchschnittlich lange fernsehen, auch mehr Zeit für die Rezeption von Videos investieren und häufiger als das Gros ihrer Altersgenossen ins Kino gehen. Umgekehrt bedeutet dies ebenfalls, dass ein geringer Femsehkonsum tendenziell mit unterdurchschnittlicher Video-Nutzung und einem selteneren 
Kino-Besuch einhergeht. Entsprechend kovariieren die durchschnittlichen Nutzungszeiten beziehungsweise -häufigkeiten in den drei hochladenden Variablen bei den Nutzem. Alle drei Variablen indizieren übergreifend Formen der rezeptiven Nutzung audiovisueller Medien. Die rotierte Hauptkomponente erklärt dabei für sich 9,3 Prozent der Varianz der analysierten Korrelationsmatrix.

\section{Hauptkomponente 4: Radio- und Musikkonsum}

Komponente 4 vereinigt die Variable zur Nutzungsdauer des Mediums Radio mit einem weiteren Item zur subjektiven Häufigkeit des Hörens von Musik. Sie hebt auf diese Weise den Konsum auditiver Medieninhalte von dem visueller Inhalte ab. Die Hauptkomponente klärt dabei für sich 8,7 Prozent der Varianz in der zugrunde liegenden Korrelationmatrix auf.

\section{Hauptkomponente 5: Comic- und Walkman-Nutzung}

Die Komponente 5 bündelt zwei Items mit Angaben zur individuellen Nutzungsdauer der Medien Comic und Walkman. Bei beiden Variablen spielt offenbar die Rezeption spaß- und unterhaltungsorientierte Inhalte durch die Jugendlichen eine zentrale Rolle. Die Hauptkomponente vereinigt dabei 8,5 Prozent der Varianz der analysierten Korrelationsmatrix auf sich.

Die fünf im Rahmen der Hauptkomponentenanalyse rekonstruierten Faktoren sollen näherungsweise die (gegenwärtige) Binnenstruktur der rezeptiven Mediennutzung bei bundesdeutschen Jugendlichen skizzieren. Methodologisch verbinden sich die Analysen dabei mit der Annahme, dass die extrahierten Komponenten voneinander unabhängig sind. D.h. konkret, Kopieren und Duplizieren, die Nutzung audio-visueller Medien, die Nutzung von Büchern, Radio- und Musikkonsum und die Nutzung anderer Printmedien aus dem Bereich von Information und Unterhaltung schließen einander nicht notwendig aus, sondern können im Einzelfall unabhängig voneinander variieren. An dieser Stelle wird zu überlegen sein, wie weit unterschiedliche Konsumptions- und Rezeptionsstile im Bereich der Mediennutzung sich eindeutig als Ausdruck der „feinen (ständischen) Unterschiede“ begreifen lassen, wie Bourdieu sie in seiner gleichnamigen Kulturanalyse der französischen Gesellschaft der 70er Jahre zugleich postulierte und konstatierte.

\subsection{Interaktive Mediennutzung}

\subsubsection{Indikatoren der interaktiven Mediennutzung}

Die interaktive Mediennutzung der Schüler wurde auf Grundlage objektivierter Zeitund Häufigkeitsangaben zur Nutzungsdauer von Computer, Internet und Handy und zur Teilnahme an Fernsehsendungen sowie darüber hinaus über Fragen zur subjektiven Häufigkeit bestimmter Formen des Mediengebrauchs er- 
fasst. Bezugspunkte waren dabei einerseits Aussagen zur allgemeinen und medienbezogenen Freizeitbeschäftigung der Jugendlichen sowie im Weiteren Angaben dazu, was sie konkret an Computer, Internet und Handy „schon einmal gemacht haben“ respektive für welche Zwecke sie diese wie häufig nutzen.

\subsubsection{Hauptkomponentenmodell zur interaktiven Mediennutzung}

Auf Grundlage der Hauptkomponentenanalyse wurde im Weiteren geprüft, wie sich die faktorielle Binnenstruktur der interaktiven Mediennutzung auf Grundlage der Befragtenangaben darstellt. Im Zuge der Analysen ergab sich, wie im Folgenden dargestellt, als beste Rekonstruktion der Daten ein Fünf- Komponenten-Modell, auf dessen Basis insgesamt 39 Prozent der Varianz in der den Analysen zugrundegelegten Korrelationsmatrix aufgeklärt werden konnten (siehe Abb. 5.2.2).

\section{Hauptkomponente 1: Konsumorientierte Mediennutzung}

Die Komponente 1 vereinigt Items zu Praktiken aus dem Bereich der EDV- und Internetnutzung, die den konkreten Nutzenaspekt dieser Medien für zum Beispiel den Gebrauch neuer Software, das Hören von Musik oder das Abwickeln von Internetgeschäften in den Vordergrund stellen. Zentral sind in diesem Zusammenhang die konsumorientierten, instrumentellen Zwecke, die der Mediennutzung zugrunde liegen. Als Items laden auf diesem Faktor: (1) „Downloading/Uploading (aus dem Internet)“, (2) „Download von Software (aus dem Internet)“, (3) „Musikdateien anhören/(aus dem Internet) herunterladen“, (4) „Etwas (aus dem Internet) bestellen, das Geld kostet“, (5) „Software installieren“, (6) „Internet nutzen“, (7) „In Datenbanken (des Internet) suchen“, (8) „Software (am Computer) installiert“. Darüber hinaus laden hier die Variablen zur individuellen Nutzungsdauer von Computer und Internet bei Jugendlichen: (9) „Computernutzung in Std. am Tag“, (10) „Internetnutzung in Std. in der Woche“. Komponente 1 erklärt, für sich betrachtet, 8,7 Prozent der Varianz in der zugrundeliegenden Korrelationsmatrix.

\section{Hauptkomponente 2: Handy-Nutzung}

Komponente 2 bündelt ausschließlich solche Items zur interaktiven Mediennutzung, die sich auf das Medium Handy beziehen. Dies sind zum einen die Items, die beinhalten, welche Funktionen Jugendliche bei ihrem Handy im Alltag nutzen (s.o.). Darüber hinaus lädt auf dem Faktor noch eines der Items, die sich auf am Handy vorgenommene Veränderungen beziehen: „Symbole oder Ruftöne auf Handy geladen“. Alle anderen Items zu vorgenommen Veränderungen konnten im Rahmen der Hauptkomponentenanalyse keine substanziellen Ladungen erzielen. Über die genannten Items tritt das Handy als eigenständige Komponente innerhalb der interaktiven Mediennutzung in Erscheinung. Sie erklärt für sich 7 Prozent der Varianz in der zugrundeliegenden Korrelationsmatrix. 


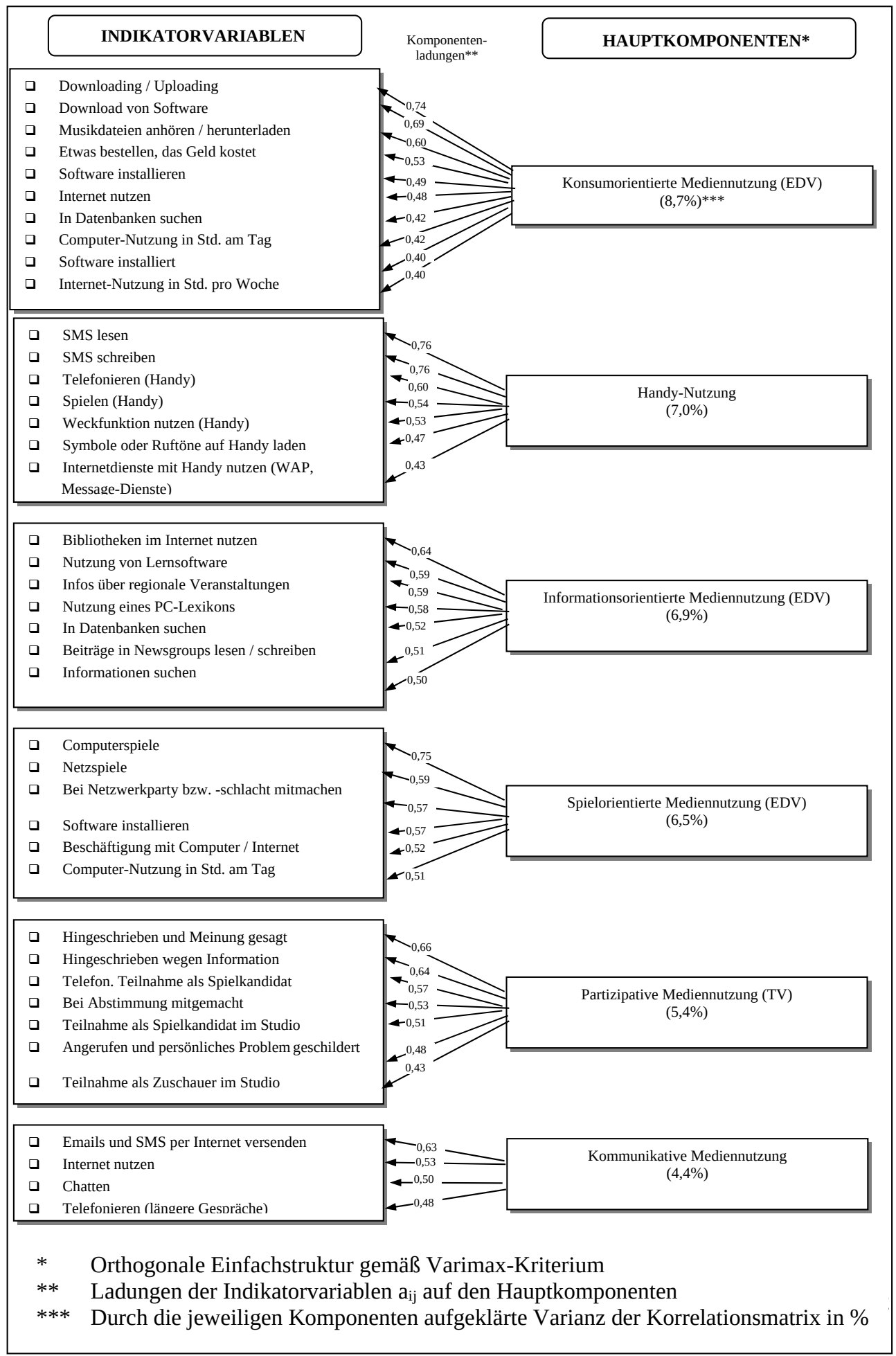

Abb. 5.2.2: Hauptkomponentenstruktur der interaktiven Mediennutzung von Jugendlichen zwischen 12 und 20 Jahren $(n=1381)$. 
Hauptkomponente 3: Informationsorientierte Mediennutzung

Auf der Komponente 3 laden sieben Items, die für die informationsorientierte Nutzung insbesondere der Neuen Medien stehen (vgl. Feierabend u. Klingler 2002). Die Items beziehen sich dabei einerseits auf die Nutzung von Informationen aus Bibliotheken und (autodidaktischer) Lernsoftware am Computer, zum anderen auf die Nutzung des Internets für Zwecke der Informationsrecherche. Es handelt sich dabei konkret um die Items: (1) „Bibliotheken im Internet nutzen“, (2) „(Recherche von) Infos über regionale Veranstaltungen“, (3) „In Datenbanken suchen“, (4) „Beiträge in Newsgroups lesen / schreiben“, (5) „Informationen suchen“. Anzumerken ist, dass das Item „In Datenbanken suchen“ ebenfalls auf Faktor 1 lädt. Dies ist als Hinweis darauf zu verstehen, dass die obige Medienaktivität durch Einflüsse eines sowohl konsumorientierten als auch informationsorientierten Nutzungsverhaltens bestimmt wird. Insgesamt erklärt die Hauptkomponente für sich 6,9 Prozent der Varianz in der zugrunde liegenden Korrelationsmatrix.

\section{Hauptkomponente 4: Spielorientierte Mediennutzung}

Auf Komponente 4 laden zum einen solche Items, die auf eine spielorientierte Nutzung von Computer und Internet verweisen. Es handelt sich dabei konkret um (1) „Spielen von Computerspielen“, (2) „(Spielen von) Netzspielen“ und (3) „Bei Netzwerkparty bzw. -schlacht mitmachen“. Zum anderen finden sich hier Items wieder, die allgemein auf die Nutzung von Computer und Internet verweisen. Es handelt sich dabei um die Items: (4) „Software installieren“, (5) „Beschäftigung mit Computer und Internet“ und (6) „Computernutzung in Std. am Tag“. Insgesamt verweist die Hauptkomponente also auf eine Spielorientierung, die sehr konkret im Kontext der Nutzung Neuer Medien steht. Sie erklärt dabei für sich 6,5 Prozent der Varianz in der zugrunde liegenden Korrelationsmatrix.

\section{Hauptkomponente 5: Partizipative Mediennutzung}

Auf dieser Komponente laden durchgängig alle Items, die sich auf verschiedene Formen der Teilnahme an Fernsehsendungen beziehen (s.o.). Sie bildet entsprechend eine partizipative Orientierung ab, die sich primär aufs Medium Fernsehen bezieht. Dabei erklärt sie, für sich betrachtet, 5,4 Prozent der Varianz in der zugrundeliegenden Korrelationsmatrix.

\section{Hauptkomponente 6: Kommunikative Mediennutzung}

Komponente 6 bündelt Variablen resp. Items, die - zum einen bezogen auf das Internet, zum anderen bezogen auf das normale Telefon - Formen der kommunikationsorientierten Mediennutzung indizieren (vgl. Feierabend u. Klingler 2002). Konkret laden hier die Items: (1) „Emails und SMS per Internet versenden“, (2) „Chatten“ und (3) „Telefonieren (längere Gespräche)“. Darüber hinaus findet sich auf diesem Faktor ebenfalls das Item „Internet 
(am Computer) nutzen“, das bereits im Kontext der konsumorientierten Mediennutzung (vgl. Faktor 1) in Erscheinung trat. Dieser Befund weist darauf hin, dass in einen nicht näher spezifizierten Internetgebrauch Aspekte einer sowohl konsumorientierten als auch kommunikativen Mediennutzung eingehen. Insgesamt gesehen erklärt Komponente 6, für sich betrachtet, 4,4 Prozent der Varianz in der zugrundeliegenden Korrelationsmatrix.

\subsection{Informative Medienkunde}

\subsubsection{Indikatoren zur informativen Medienkunde}

Das informativ-medienkundliche Wissen der Schüler wurde über Wissensfragen zu verschiedenen medienbezogenen Themengebieten erfasst. Thematisch wurden dabei unterschieden:

a) Fragen nach Autoren oder nach Vertretern medienspezifischer Inhalte, z.B.

- Weißt du, wer das Buch „Der Herr der Ringe“ geschrieben hat?

- Welcher Musiker hat den Reggae weltberühmt gemacht?

- Wer hat die beweglichen Metallbuchstaben für den Buchdruck erfunden?

b) Fragen zu Spezifika einzelner Medien, wie etwa

- Bei welchem Sender wird die Sendung „Gute Zeiten - Schlechte Zeiten“ ausgestrahlt?

- Wie nennt man Großkinos, die sich durch vielfältige Programme, technische Perfektion und ein umfangreiches Serviceangebot (Getränke, Speisen etc.) auszeichnen?

- Was sind „Mangas“?

c) Fragen zu den gegebenen Strukturen des Mediensystems

- Welches ist derzeit das am meisten verbreitete private Videosystem der Welt?

- Wodurch finanziert sich der Fernsehsender RTL?

- Was ist ein Volontariat?

Die in die Studie einbezogenen Jugendlichen beantworteten insgesamt 27 Fragen zur informativen Medienkunde. Davon mussten neun Fragen für die weiteren Analysen ausgeschieden werden, da sie die Mindestkriterien für die Itemschwierigkeit und die Trennschärfe nicht erfüllten (siehe auch Abschnitt 4.1). Von den verbliebenen 16 Items wiesen insgesamt 14 substantielle Ladungen auf den beiden interpretierbaren Hauptkomponenten zum Medienwissen auf (siehe Abschnitt 5.3.2). 


\subsubsection{Hauptkomponentenmodell zur informativen Medienkunde}

Auf Grundlage der Hauptkomponentenanalyse wurde im Weiteren geprüft, wie sich die Binnenstruktur des informativen Medienwissens auf Grundlage der Befragtenangaben darstellt. Im Zuge der Analysen ergab sich, wie im Folgenden dargestellt, als beste Rekonstruktion der Daten ein Zwei-Komonenten-Modell, auf dessen Basis insgesamt 34,3 Prozent der Varianz in der den Analysen zugrundegelegten Korrelationsmatrix aufgeklärt werden konnten (siehe Abb. 5.3.2).

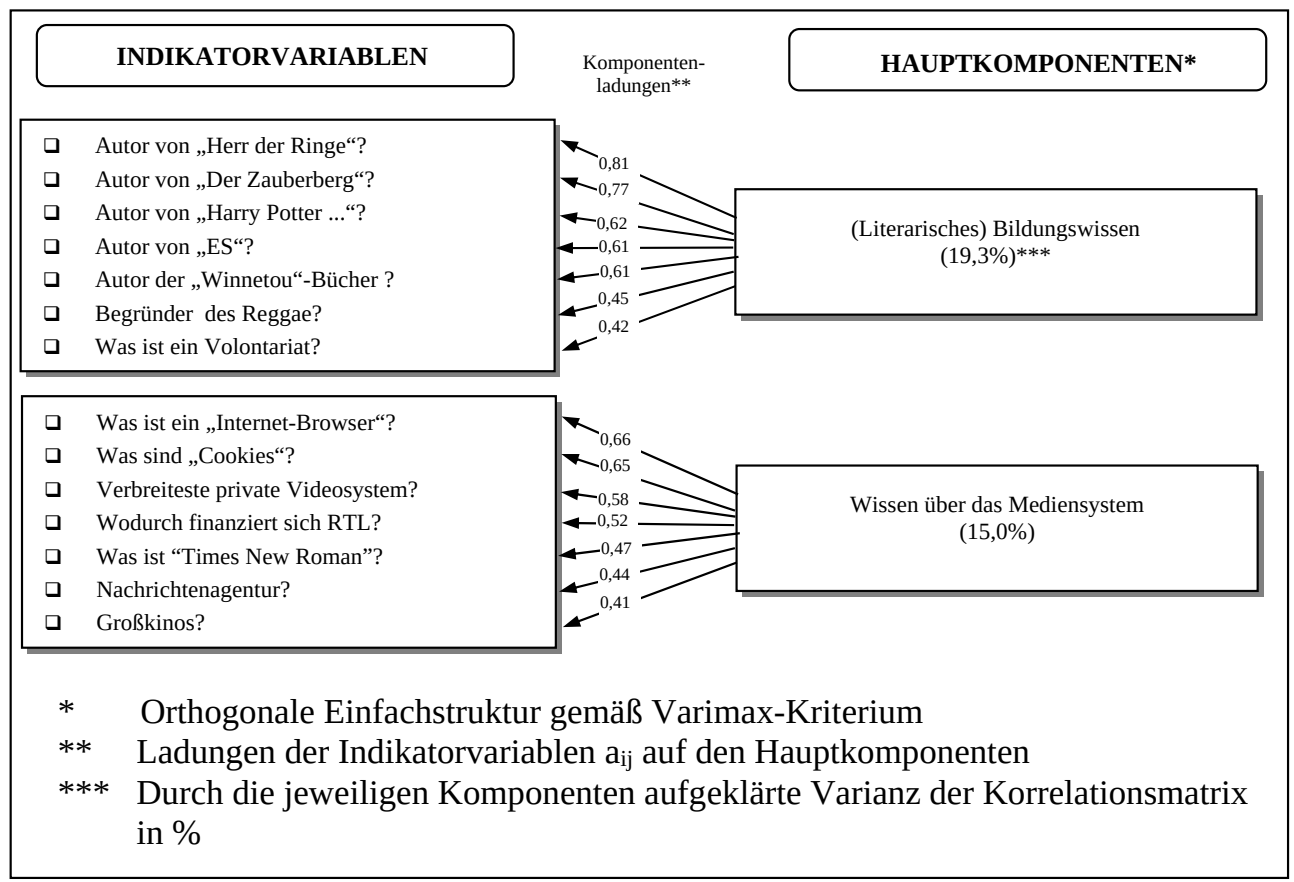

Abb. 5.3.2: Hauptkomponentenstruktur der informativen Medienkunde von Jugendlichen zwischen 12 und 20 Jahren $(n=1867)$

\section{Hauptkomponente 1: Literarisches Bildungswissen}

Die Komponente 1 vereinigt Fragen zu vornehmlich literarischen Wissensinhalten auf sich. Es handelt sich dabei zum einen um Fragen nach Buchautoren: (1) „Autor von ,Herr der Ringe““, (2) „Autor von ,Der Zauberberg““, (3) „Autor von ,Harry Potter““ (4) „Autor von ,Es““ und (5) „Autor von Winnetou““. Zum anderen findet sich hier ebenfalls die Frage zu einem berühmten Vertreter des Reggae (6), eine Frage, deren Beantwortung nicht primär „Buchwissen“, sondern einen Kenntnishorizont in dem Bereich der Popularmusik voraussetzt. Das Item (7) auf diesem Faktor, die Frage „Was ist ein Volontariat?, ist ebenfalls nicht direkt auf literarisches Wissen bezogen, hat aber einen starken Bezug zum Printmediensektor. Die Hauptkomponente er- 
klärt, für sich betrachtet, 19,3 Prozent der Varianz in der den Analysen zugrunde liegenden Korrelationsmatrix.

Hauptkomponente 2: Wissen über das Mediensystem

Komponente 2 fasst im Weiteren solche Fragen zusammen, die eher sachbezogene Themen beinhalten. Es handelt sich dabei einerseits um technisch ausgerichtete Fragen zu EDV und Internet: (1) „Was ist ein Internet-Browser?“, (2) „Was sind Cookies?“ und (3) „Was ist Times New Roman?“ Daneben sind hier aber auch solche Fragen gebündelt, die eher ein Wissen über einzelne Medien oder Medieninstitutionen beinhalten, so etwa (4) die Frage nach dem am meisten verbreiteten privaten Videosystem oder (5) die Frage „Wodurch finanziert sich der Fernsehsender RTL?“. Ebenfalls auf Sachwissen über das Mediensystem rekurrieren die auf diesem Faktor ladenden Items: (6) „Was ist eine Nachrichtenagentur?“ und (7) „Wie nennt man Großkinos, die sich u.a. durch ein umfangreiches Serviceangebot auszeichnen?“ Hauptkomponente 2 erklärt für sich 15 Prozent der Varianz in der den Analysen zugrunde liegenden Korrelationsmatrix.

Die jeweils sieben Items, die auf den beiden Hauptkomponenten substantielle Ladungen aufweisen, lassen sich damit zu zwei Skalen zusammenfassen, um die beiden Konstrukte „Literarisches Bildungswissen“ und „Wissen über das Mediensystem“ zu messen. Die beiden aus jeweils sieben Items bestehenden Skalen erfassen die Konstrukte hinreichend zuverlässig und erlauben somit zumindest Gruppenvergleiche zwischen Jugendlichen anzustellen, da Cronbachs Alpha für die erste Skale 0,78 und für den zweiten Index 0,68 beträgt (siehe auch Kapitel 4.1).

\subsection{Kreative Mediengestaltung}

\subsubsection{Indikatoren zur kreativen Mediengestaltung}

Die kreative Mediengestaltung der Schüler wurde über Fragen zu der von ihnen berichteten Häufigkeit bestimmter Formen des Mediengebrauchs erfasst. Bezugspunkte waren dabei einerseits Aussagen zur allgemeinen und medienorientierten Freizeitbeschäftigung der Jugendlichen sowie im Weiteren Angaben dazu, welche gestalterischen Dinge sie insbesondere im Umgang mit Neuen Medien „schon einmal gemacht haben“ respektive für welche Zwecke sie diese wie häufig nutzen.

\subsubsection{Hauptkomponentenmodell zur kreativen Mediengestaltung}

Auf Grundlage der. Hauptkomponentenanalyse wurde im Weiteren geprüft, wie sich die faktorielle Binnenstruktur der kreativen Mediengestaltung auf Grundlage der Befragtenangaben darstellt (siehe Abb. 5.4.2). 


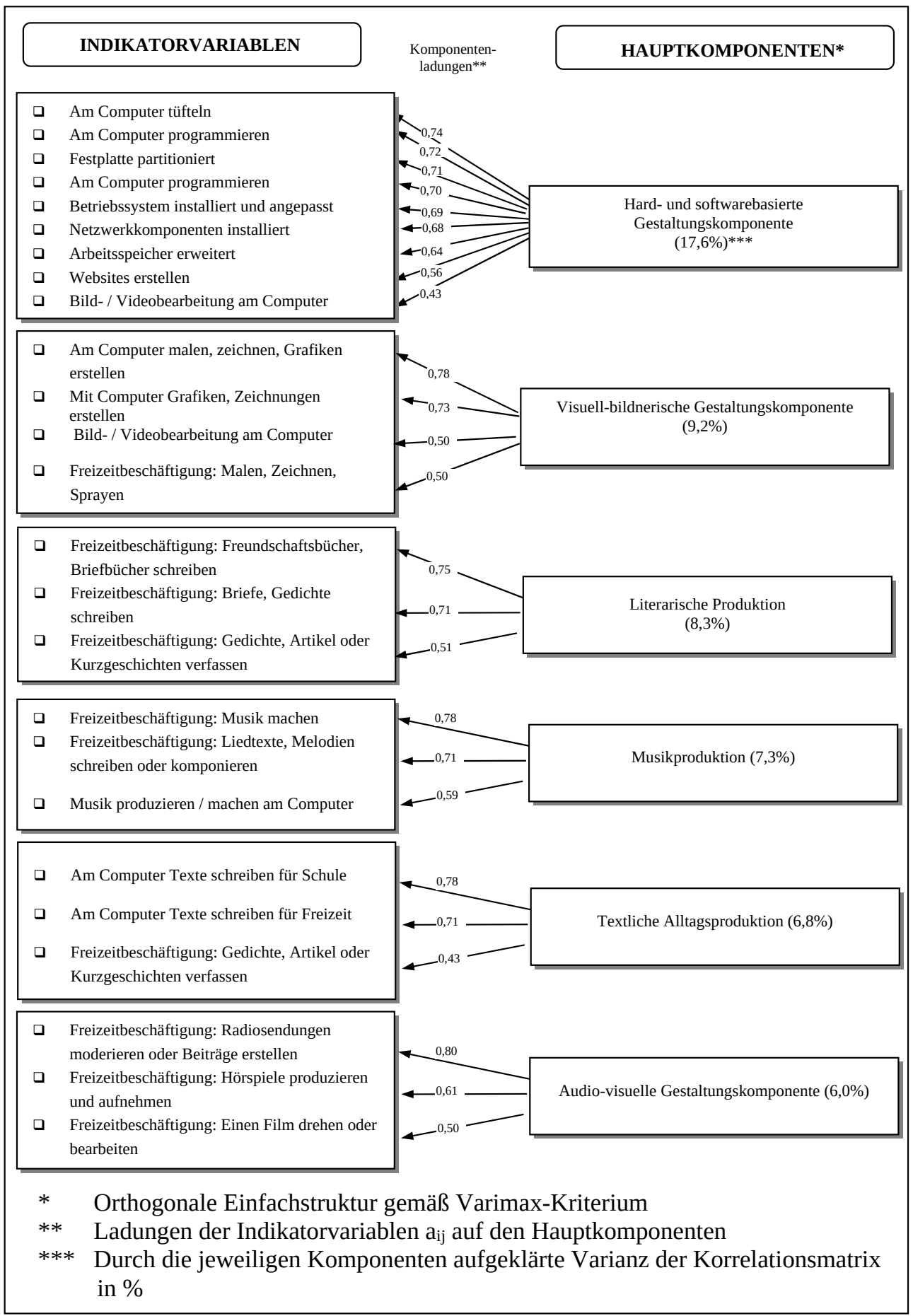

Abb. 5.4.2: Hauptkomponentenstruktur der Mediengestaltung von Jugendlichen zwischen 12 und 20 Jahren $(n=1923)$. 
Im Zuge der Analysen ergab sich als beste Rekonstruktion der Daten ein Sechs-Komponenten-Modell, auf dessen Basis insgesamt 55 Prozent der Varianz in der den Analysen zugrundegelegten Korrelationsmatrix aufgeklärt werden konnten (siehe Abb. 7.3.2.1).

\section{Hauptkomponente 1: Hard- und softwarebasierte Gestaltungskomponente}

Auf der Komponente 1 laden insbesondere solche Items, die sich auf allgemein gestalterische Arbeiten am Computer beziehen. Dabei stehen hard- und softwarebezogene Gestaltungsaktivitäten nebeneinander. Als hardwarebezogene Gestaltungsaktivitäten laden hier die Items: (1) „Am Computer tüfteln“ und (2) „Arbeitsspeicher erweitert“. Im Weiteren laden als softwarebezogene Gestaltungsaktivitäten: (3) „Am Computer programmieren“, (4) „Festplatte partitioniert“, (5) „Betriebssystem installiert und angepasst“, (6) „Netzwerkkomponenten installiert“, (7) „Websites erstellen“, (8) „Bild-/ Videobearbeitung am Computer“.. ${ }^{3}$ Der Faktor erklärt dabei für sich gesehen 17,6 Prozent der Varianz in der zugrundeliegenden Korrelationsmatrix und ist im Vergleich zu allen weiteren Hauptkomponenten besonders varianzstark.

\section{Hauptkomponente 2: Visuell-bildnerische Gestaltungskomponente}

Komponente 2 bündelt solche Items, die auf grafisch-zeichnerische und bildbezogene Gestaltungsaktivitäten verweisen. Es laden hier zum einen solche Items, die auf entsprechende Aktivitäten am Computer Bezug nehmen: (1) „Am Computer malen, Zeichnen, Grafiken erstellen“, (2) „Mit Computer Grafiken, Zeichnungen erstellen“, (3) Bild-/ Videobearbeitung am Computer“. Zum anderen lädt hier auch das Item „Freizeitbeschäftigung: Malen, Zeichnen, Sprayen“ (4), das über das Arbeiten mit edv-spezifischen Anwendungen hinaus auf zeichnerisch-bildnerische Aktivitäten verweist. Da Item (3) dieses Faktors ebenfalls eine Ladung auf der Komponente 1 des vorliegenden Modells aufweist, ist davon auszugehen, dass beide Hauptkomponenten die entsprechende Medienaktivität beeinflussen. Der vorliegende Faktor erklärt 9,2 Prozent der Varianz in der den Analysen zugrundeliegenden Korrelationsmatrix.

\section{Hauptkomponente 3: Literarische Produktion}

Komponente 3 bündelt solche Items, die auf das Schreiben resp. Verfassen persönlicher oder auch subjektbezogener Texte Bezug nehmen. Es handelt sich dabei um die Items: (1) „Freizeitbeschäftigung: Freundschaftsbücher, Briefbücher schreiben“, (2) „Freizeitbeschäftigung: Briefe, Gedichte schreiben“ und (3) „Freizeitbeschäftigung: Gedichte, Artikel oder Kurzgeschichten

3 Dem Faktor wurde im Zuge der Hauptkomponentenanalysen ebenfalls das Item „Zusatzgeräte installiert“ zugewiesen. Das Item konnte auf dieser Komponente jedoch keine substantielle Ladung $(r \geq 0,40)$ erzielen. 
verfassen“. Die Hauptkomponente bezieht sich dabei primär auf inhaltliche Aspekte der Textproduktion. Sie erklärt dabei, für sich betrachtet, 8,3 Prozent der Varianz in der zugrundeliegenden Korrelationsmatrix.

\section{Hauptkomponente 4: Musikproduktion}

Komponente 4 bündelt Items, die sich auf kreative Aktivitäten im Kontext des Musikmachens resp. der Musikproduktion beziehen. Es laden auf diesem Faktor entsprechend die Items: (1) „Freizeitbeschäftigung: Musik machen“, (2) „Freizeitbeschäftigung: Liedtexte, Melodien schreiben oder komponieren“ und (3) „Musik produzieren/machen am Computer““. ${ }^{4}$ Die Hauptkomponente 4 erklärt, für sich betrachtet, 7,3 Prozent der Varianz in der zugrundeliegenden Korrelationsmatrix.

\section{Hauptkomponente 5: Textliche Alltagsproduktion}

Komponente 5 vereinigt solche Items, die sich statt auf inhaltliche, eher auf schreibbezogene Aspekte der Textproduktion beziehen. So laden hier die Items: (1) „Am Computer Texte schreiben für die Schule“ und (2) „Am Computer Texte schreiben für Freizeit“. Darüber hinaus lädt auf diesem Faktor, allerdings mit relativ schwacher Ladung, das Item „Freizeitbeschäftigung: Gedichte, Artikel oder Kurzgeschichten verfassen“, das bereits auf dem Faktor 3 zur literarischen Produktion in Erscheinung trat. Dies indiziert, dass beide Hauptkomponenten als Faktoren textbezogener Gestaltungsaktivitäten das genannte Item beeinflussen. Die Komponente 5 erklärt dabei, für sich betrachtet, 6,8 Prozent der Varianz in der zugrundeliegenden Korrelationsmatrix.

\section{Hauptkomponente 6: Audio-visuelle Gestaltungskomponente}

Auf der Komponente 6 laden solche Items, die sich auf kreativ-gestalterische Aktivitäten im Umgang mit auditiven und visuellen Medien beziehen. Es handelt sich dabei um die Items: (1) „Radiosendungen moderieren oder Beiträge erstellen“, (2) „Hörspiele produzieren und aufnehmen“ und (3) „Einen Film drehen/bearbeiten“. Die Hauptkomponente erklärt 6 Prozent der Varianz in der zugrundeliegenden Korrelationsmatrix.

Wichtig ist an dieser Stelle herauszustellen, dass die vorgestellten Komponenten kreativ-gestalterischen Medienhandelns - analog zu den methodologischen Prämissen der Hauptkomponentenanalyse - als voneinander unabhängig zu betrachten sind. Das heißt, illustriert an einem Beispiel, dass etwa gestalterische Aktivitäten im Bereich der Musikproduktion gestalterische Aktivitäten in dem Bereich literarischer Textproduktion weder ausschließen noch implizieren und deshalb, bezogen auf das Kompetenzprofil von Ju-

4 Durch die Hauptkomponentenanalyse wurde zwar auch das Item „Rufton für das Handy komponieren“ diesem Faktor zugeordnet. Es konnte dabei aber keine substantielle Ladung $(r \geq 0,40)$ auf der Komponente erzielen. 
gendlichen, in unterschiedlichen Variationen Vorkommen können. Ein wichtiges Ergebnis der dokumentierten Analysen besteht schließlich darin, dass ein eigenständiger Faktor hard- und softwarebasierter Gestaltungsaktivitäten und eine Hauptkomponente der visuell-bildnerischen Produktion - realisiert im Wesentlichen über die Möglichkeiten, die das Medium Computer zu diesem Zwecke bietet - in Erscheinung treten. Dieser Befund verweist auf die immense Bedeutung, die Neue Medien auch im Bereich kreativgestalterischen Medienhandelns für Jugendliche haben.

\section{Fazit und Ausblick}

\subsection{Fazit}

Die Hauptkomponentenanalysen liefern für verschiedene Bereiche des Medienhandelns, die sich am Bielefelder Medienkompetenz-Modell mit seinen Dimensionen der Mediennutzung, Medienkunde, Mediengestaltung und Medienkritik orientierten, intepretierbare faktorielle Binnenstrukturen, die es erlauben, Hauptquellen der Unterschiede, welche Jugendliche in ihren Medienaktivitäten zeigen, zu rekonstruieren. Darüber hinaus lässt sich für die verschiedenen Felder des Medienhandelns nicht eine eindimensionale, sondern eine mehrdimensionale Ordnungsstruktur ableiten, die mit den empirisch erhobenen Daten verträglich ist und zugleich den empirischen Gehalt des Modells verdeutlicht.

1) Es lassen sich interpretierbare faktorielle Binnenstrukturen für die folgenden Unterdimensionen des Bielefelder Medienkompetenz-Modells aus den Umfragedaten rekonstruieren:

\section{Rezeptive Mediennutzung}

- Kopieren und Duplizieren

- Nutzung informations- und unterhaltungsorientierter Printmedien

- Nutzung audio-visueller Medien

- Radio- und Musikkonsum

- Comic- und Walkman-Nutzung

\section{Interaktive Mediennutzung}

- Konsumorientierte Mediennutzung (EDV)

- Handy-Nutzung

- Informationsorientierte Mediennutzung (EDV)

- Spielorientierte Mediennutzung (EDV)

- Partizipative Mediennutzung (TV)

- Kommunikative Mediennutzung 
Informative Medienkunde

- (Literarisches) Bildungswissen

- Wissen über das Mediensystem

Kreative Mediengestaltung

- Hard- und softwarebasierte Gestaltungskomponente

- Visuell-bildnerische Gestaltungskomponente

- Literarische Produktion

- Musikproduktion

- Textliche Alltagsproduktion

- Audio-visuelle Gestaltungskomponenten

2) Diese faktoriellen Binnenstrukturen bilden zugleich empirisch gestützte Klassifikationen jugendlichen Medienhandelns.

3) Sowohl die Ergebnisse der univariaten Analysen (über die in diesem Beitrag nicht berichtet wird) als auch der multivariaten Analysen zur interaktiven Mediennutzung zeigen die inzwischen bestehende massive Durchdringung jugendlicher Lebenswelten mit den Neuen Medien „Computer“, „Internet“ und „Handy“ (vgl. Vogelgesang 1994). Ihre Veralltäglichung und die zu ihrer Nutzung erforderlichen Aneignungsprozesse zur Bildung entsprechenden kulturellen Kapitals scheinen weit fortgeschritten zu sein.

4) Die Möglichkeit der Rekonstruktion der drei Hauptkomponenten „Hard- und softwarebasierte Gestaltungskomponente“, „Visuell-bildnerische Gestaltungsaktivitäten“ und „Textliche Alltagsproduktion“ zeigt die große Bedeutung, die die Neuen Medien für die gegenwärtige Generation der Jugendlichen auch im Bereich des kreativ-gestalterischen Medienhandelns besitzen.

\subsection{Ausblick}

In einem nächsten Auswertungsschritt werden die Komponentenwerte der befragten Jugendlichen für jede Hauptkomponente getrennt berechnet. Der Faktorwert eines Jugendlichen bezeichnet seine Position auf der betreffenden Komponente. Er informiert darüber, wie stark die in einer Hauptkomponente zusammengefassten Merkmale bei diesem Jugendlichen - im Vergleich zu den anderen Jugendlichen in der Stichprobe - ausgebildet sind. Die Komponentenwerte lassen sich damit als Ausprägungen interpretieren, welche die Jugendlichen auf den einzelnen Hauptkomponenten erreichen. Diese varianzstarken beziehungsweise erklärungsmächtigen Variablenbündel, die auf einer höheren Abstraktionsstufe als die Eingangsvariablen hegen und einen höheren Verallgemeinerungsgrad besitzen, bilden die Ausgangsdaten für die Durchführung von Clusteranalysen zur Entwicklung einer empirisch gestützten Typologie des Medienhandelns Jugendlicher. 


\section{Literaturverzeichnis}

Baacke, D.: Medienkompetenz - Begrifflichkeit und sozialer Wandel. In: Rein, A. von (Hrsg.): Medienkompetenz als Schlüsselbegriff. Bad Heilbrunn (1996).

Baacke, D.: „Medienkompetenz“: theoretisch erschließend und praktisch folgenreich. In: Medien und Erziehung, 1/1999, S. 7-12.

Bortz, J.: Statistik für Sozialwissenschaftler. 5. Aufl. Berlin 1999.

Bortz, J./ Döring, N.: Forschungsmethoden und Evaluation für Human- und Sozialwissenschaften. 3. Aufl. Berlin 2002.

Bourdieu, P.: Ökonomisches Kapital-Kulturelles Kapital-Soziales Kapital. In: Die verborgenen Mechanismen der Macht. Schriften zu Politik \& Kultur 1 (Hg. von M. Steinrücke). Hamburg 1997, S. 49-79.

Cureton, E. E./ D’Agostino, R. B.: Factor Analysis: An Applied Approach. Hillsdale. New Jersey 1983.

Diekmann, A.: Empirische Sozialforschung. Grundlagen, Methoden, Anwendungen. Reinbek bei Hamburg 1995.

Feierabend, S./ Klingler, W.: Medien- und Themeninteressen Jugendlicher. Ergebnisse der JIM-Studie 2001 zum Medienumgang 12- bis 19-Jähriger. In: Media Perspektiven. 1/2002, S. 9-21.

Gerhards, M./ Klingler, W.: Jugend und Medien: Fernsehen bleibt dominierend. Nutzung und Bedeutung des Fernsehens für Jugendliche im Jahr 2000. In: Media Perspektiven. 2/2001, S. 65-74.

Jackson, J. E.: A User's Guide to Principal Components. New York 1991.

Jolliffe, I. T.: Principal Component Analysis. New York 1986.

Katz, E./ Blumler, J. G. (Hrsg.): The Uses of Mass Communications. Current Perspectives on Gratification Research. Beverly Hills/London 1974.

Krzanowski, W. J./ Marriott, F. H. C.: Multivariate Analysis, Part 1: Distributions, Ordination and Inference; Part 2: Classification, Covariance Structures and Repeated Measurements. London 1994 u. 1995.

Tabachnick, B.G./Fidell, L.S.: Using Multivariate Statistics. 3rd. Edition. New York 1996.

Treumann, K.P./Baacke, D./Haacke, K./Hugger, K.U./Vollbrecht, R.: Medienkompetenz im digitalen Zeitalter. Wie die neuen Medien das Leben und Lernen Erwachsener verändern. Opladen 2002.

Vogelgesang, W.: Jugend- und Medienkulturen. Ein Beitrag zur Ethnographie medienvermittelter Jugendwelten. In: Kölner Zeitschrift für Soziologie und Sozialpsychologie. 46, 1994, 3, S. 464491. 